\title{
Unsupervised zoning of cultivation areas with similar cultivation pattern in Golestan province based on the vegetation products of MODIS sensor
}

\author{
F. Youssefi ${ }^{1 *}$, M.J. Valadan zoej ${ }^{2}$, A. Safdarinezhad ${ }^{3}$, M.R Sahebi ${ }^{4}$ \\ ${ }^{1}$ Dept. of Photogrammetry and remote sensing, K.N.Toosi University, Valiasr Street, Tehran, Iran - youssefi@email.kntu.ac.ir \\ ${ }^{2}$ Dept. of Photogrammetry and Remote sensing, K.N.Toosi University, Valiasr Street, Tehran, Iran - valadanzouj@kntu.ac.ir \\ ${ }^{3}$ Dept. of Geodesy and surveying Engineering, Tafresh University, Tafresh, Iran - safdarinezhad@ tafreshu.ac.ir \\ ${ }^{4}$ Dept. of Photogrammetry and remote sensing, K.N.Toosi University, Valiasr Street, Tehran, Iran - sahebi@.kntu.ac.ir
}

Commission VI, WG VI/4

KEY WORDS: Cultivation area - Cultivation pattern - Clustering - Sequential maximum angle convex cone endmember extraction - Spectral angle mapper

\begin{abstract}
:
The estimation of cultivation area and categorizing the agricultural product types is one of the prerequisites for achieving sustainable development in the agricultural studies. In this study, an unsupervised zoning the cultivation areas with the same cultivation pattern in Golestan province is on the agenda. Therefore, due to wide spatial range, high temporal resolution and easy access of 16-day products of the vegetation of the MODIS sensor which acquired in a year (From November 2017 to October 2018), these images are used in this research. In the proposed method, after the generating of NDVI vegetation time series as a hyper-cube and separating farmlands' boundaries in Golestan province using the land-use map; the Sequential Maximum Angle Convex Cone (SMACC) endmember extraction algorithm and the maximum number of product variation using the statistical information of the region (Obtained from the statistics centre of Iran) are used to extract endmembers of the hyper-cube. In the following, the timing responses of the NDVI, identified as endmembers, will be refined in the second phase. In this process, identifying and eliminating noise signals (unrelated to cultivating patterns) and integrating the same cultivating patterns will be on the agenda. At the last stage of the proposed method and after refinement of the endmembers, the hyper-cube is clustered by Spectral Angle Mapper (SAM) algorithm and the mapping of regions with the same cultivation pattern is produced. In the proposed method, the zoning of agricultural land is based solely on the statistical knowledge of the variety of cultivation and the results have led to the production of interconnected spatial parts. This is consistent with the reality of the spatial occurrence of similar cultivating patterns in a geographic area. On the other hand, the visual comparison of results with large scale satellite images illustrates that there is a significant relationship between clustering results and ground truth in terms of cultivating pattern. Obviously, such products can be used as initial layers of information to produce the results of a supervised classification with the aim of applying the cultivation area of a variety of agricultural products.
\end{abstract}

\section{INTORODUCTION}

Obviously, the basis of proper planning and management of agricultural resources is based on having accurate and timely statistics and information on agricultural capacities. Today, remote sensing technology is recognized as a reliable and cost-effective approach in this field. Remote sensing satellites enable specialists to extract different information by providing varied data from cultivation areas (Abdolahzadeh and Nasiri, 2007). Therefore, the use of remote sensing technology in agricultural studies has grown in recent years. However, there are still numerous challenges in this field that focus on research.

Two of the practical indices used to distinguish crops in remote sensing technology is phenological differences and crop calendars (Yan et al., 2015). Accordingly, the production of time-series and consequently their analysis is one of the common approaches to extracting crops maps. So far, various studies have been conducted on the use of satellite imagery for land-use mapping. In the meantime, time series data acquired by
MODIS sensor have attractive advantages such as convenient temporal resolution and cost-free for the Earth's surface observation and thus has been greatly welcomed and utilized in current research. For example, (Sanaeinejhad et al., 2007) used 16-day NDVI images to estimate wheat cultivation in Khorasan Razavi province. They first classified the study area into four classes (desert, wheat field, mountain and vegetation) using a supervised classification method. Then, by using a comparative analysis based on spectral data and calculated NDVI, the wheat crop map and its area were determined. (Wardlow et al., 2007) investigated the temporal trend of vegetation indices to classify large fields of agriculture including alfalfa, corn, soybeans and wheat using MODIS $250 \mathrm{~m}$ vegetation index data and based on the different temporal trends of EVI and NDVI, different classes are separated. The results showed that the separation of cereals during the ripening time of products was more significant in terms of crop growth period. (Zhang et al., 2010) observed similar phenological trends throughout the growing seasons of crop varieties, using the NDVI product of the MODIS and field measurements. The results indicated that the

\footnotetext{
* Corresponding author
} 
farmlands had distinct characteristics in terms of average values of vegetation index in different periods, which provided the importance of developing a new classification method by using probability distribution of vegetation index instead of using average vegetation index. (Brown et al., 2013) attempted to classify the EVI and NDVI vegetation products of MODIS in Brazil. They trained the decision tree supervised classification algorithm and classified the MODIS vegetation products using a ground truth dataset collected over 5 years to define endmember pixels. The need to collect costly ground data is a downside to the method proposed by Brown. (Li et al., 2015) using a threestep process including selection of optimum features, objectbased image segmentation analysis and finally classification based on decision tree algorithm. They have analysed different crop types using MODIS and Landsat images. They performed this study using an improved time series in the form of 26 NDVI image layers and 5 NDVI time series indices (TSIs). In their research, the effect of noise due to field heterogeneity and spectral variability has been reduced due to apply the objectbased analysis. (Yan et al., 2015) developed various phenological indices using time series obtained from MODIS vegetation products including EVI and NDVI. Then, the amplitude and phase information of the harmonic components of the time-spectral response of the samples in the time series are estimated using the Fourier transform. The separability of eight vegetation cover types plus water and built-up areas was then analysed using phenological metrics, and amplitude and phase of harmonic components. The results showed that Fourier transforms of NDVI and EVI time series led to the first four harmonic components (including component 0 , average annual NDVI, and EVI) that captured the phenological characteristics of the cover types. Also, compared to those using only NDVI, the separability values of the classes using NDVI, amplitude, and phase increased, implying the potential improvement of classification. (Chen et al., 2015) developed a new approach using MODIS NDVI time series data to identify crops including soybean, cotton and maize. They first enhanced NDVI time series data by deleting cloud pixels using local interpolation algorithm and then identified and selected the best time periods and phenological parameters to better discriminate crops. They first enhanced NDVI time series data by deleting cloud pixels using local interpolation algorithm and then identified and selected the best periods and phenological parameters to better discriminate crops. Next, they generated temporal indices for crops to extract the cultivation patterns of each crops using NDVI time series data and finally classify the cultivation patterns using decision tree classifier and temporal indices.

Climatic variation in Iran has led to the same pattern of cultivating in different geographical regions. In this regard, one of the prerequisites for developing algorithms to separate agricultural products is the method based on cultivation pattern and agronomic calendar. In this study, Based on the background of the research, as well as the increased financial costs of field measurements due to different crops with different harvesting time in Iran, this study presents an unsupervised method to classify farmland. In the following, the proposed method and the results are discussed.

\section{PROPOSED METHOD}

In this research, in order to separate the same cultivation area, a hyper-cube of NDVI images is first created. Because the NDVI image shows any vegetation, such as forests, shrubs, gardens, etc., farmlands should first be separated using existing maps from other areas. These maps have already been developed using remote sensing data and during the classification process. In the following, using the reports published by the Iranian Statistics centre (ISC) about the diversity of crops and their annual cultivation pattern, the number of product varieties and the cultivation time of each product are obtained. Then, it is necessary to extract endmembers of the hyper-cube of NDVI images in order to cluster the hyper-cube image and separating the regions with the same cultivation pattern. To obtain this result, the SMACC algorithm has been used. This algorithm uses a convex cone model to display vector data. In this iterative method, the components are selected directly from the data set. In this method, the selection of endmembers initially starts with one member, and then other members are added more and more. Then, the timing responses of NDVI that are identified as endmembers are refined. In this refinement, noise signals that are unrelated to the cultivation patterns have been identified and eliminated. On the other hand, cultivation patterns that are similar in terms of timing responses are integrated with each other (the same cultivation patterns are used to refer to cases where the cropping mechanism is similar and only the time of its start is made with little difference. This subject depends on the decision to start planting by farmers in the permitted periods of planting). In the last step, the hypercube of NDVI images are clustered by the SAM algorithm and the map of the separated farmlands with the same cultivation pattern is generated.

\section{RESULTS AND DISCUSION}

In the upcoming research, by using 16-day products of the vegetation of the MODIS sensor which acquired in a year from Golestan province, a time series was created in the form of a hyper-cube image. Each band of this image is equivalent to one time from the time series of NDVI images. By using land-use maps, agricultural areas are distinguished from other land cover, also, the diversity of the province's agricultural products and the agronomic calendar of each of them are provided using the annual reports of the Iranian Statistics Centre (ISC) (Figure 1).

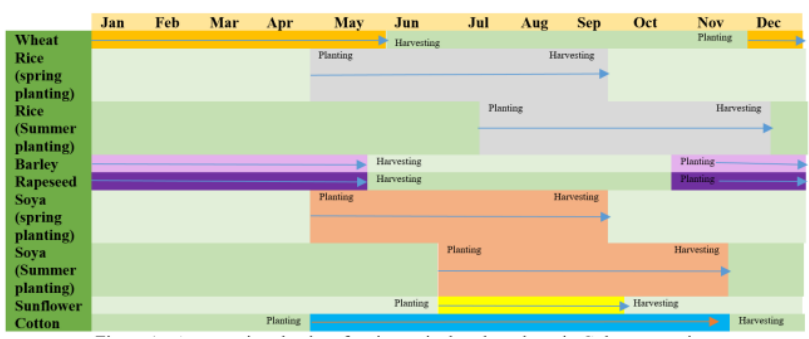

Figure 1. Agronomic calendar of major agricultural products in Golestan province

It should be mentioned that the agronomic calendar in comparison with the cultivation pattern is not necessarily the same and more than one agricultural products might be grown on a farmland within a year .Accordingly, the variety of cultivation patterns is generally more diverse than agricultural products, and therefore the number of endmembers is higher than the number of crops.

After determining the number of crop varieties, endmembers are extracted using the SMACC algorithm from the hyper-cube image and the timing responses of the NDVI, identified as endmembers, will be refined. (Figure 2). 


\section{REFERENCES}

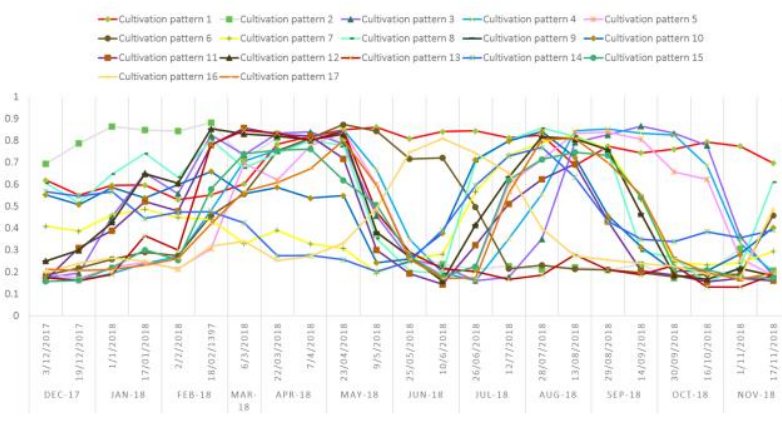

Figure 2. Major cultivation pattern of agricultural products in Golestan province

In the process of refinement, two main steps are taken. The first step is to eliminate signals that are meaningless in terms of greenness. In this process, the NDVI time series' signals that have these conditions: 1- Sustainability throughout the crop year; 2- Intermittent and rapid behavior in short-term intervals; and 3. Maximum non-compliance of greenness with the expected time in Table 1; are deleted. The second step is to integrate the signals that have the same general behavior, and they only have a slight difference in planting and harvesting times. These signals are indicative of similar cultivation patterns, with which farmers have begun to plant.

In the final step, after refinement of endmembers, the hypercube image is clustered by the SAM algorithm according to the number of identical culture patterns (Fig. 3). At this stage, the threshold of the angle which is used in SAM algorithm for nonclustering of noise samples is considered.

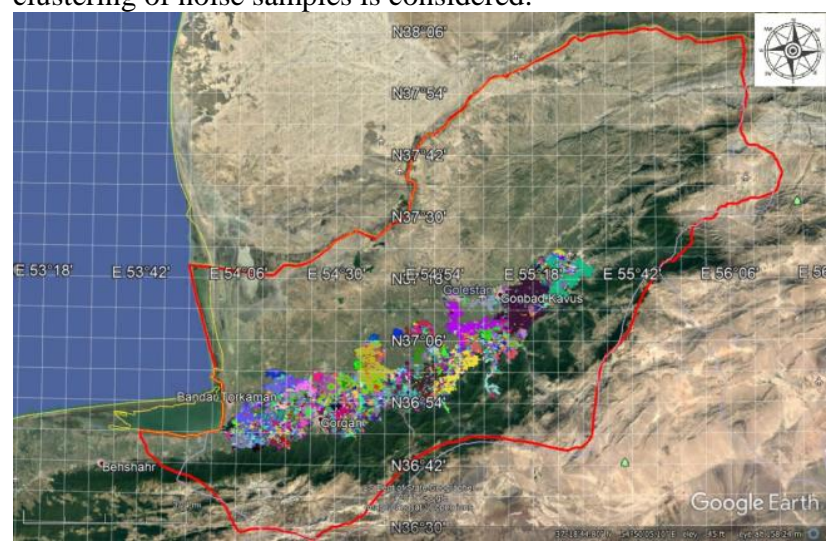

Figure 3. Clustering the study of area based on the same cultivation patterns

The results are evaluated by visual comparison of large-scale satellite imagery, and this assessment confirms a good relationship between the results of the clustering of the proposed method and the ground truth. In the proposed method, it is only by using statistical knowledge of the diversity of crops in the area of study, the same cultivation patterns can be identified and the user, without field surveying, can obtain the location of the same cultivation patterns. Ultimately, If the classification is necessary, only enough to collect the train data as many as the number of existing clusters.
Abdolahzadeh, M., Nasiri, M.B. 2007. Determination of potato cultivation area in Borujen city using IRS-P6 time series. Geomatic Conference 2007, National Cartographic Centre, Tehran, Iran.

Brown, J.C., Kastens, J.H., Coutinho, A.C., de Castro Victoria, D. and Bishop, C.R., 2013. Classifying multiyear agricultural land use data from Mato Grosso using time-series MODIS vegetation index data. Remote Sensing of Environment, 130, pp.39-50.

Chen, Y., Lu, D., Moran, E., Batistella, M., Dutra, L.V., Sanches, I.D.A., da Silva, R.F.B., Huang, J., Luiz, A.J.B. and de Oliveira, M.A.F., 2018. Mapping croplands, cropping patterns, and crop types using MODIS time-series data. International journal of applied earth observation and geoinformation, 69, pp.133-147.

Li, Q., Wang, C., Zhang, B. and Lu, L., 2015. Object-based crop classification with Landsat-MODIS enhanced time-series data. Remote Sensing, 7(12), pp.16091-16107.

Sanaeinejhad, S.H., Sadr AbadiHaghighat, R., Klarastaghi, K., ShahTahmasbi, A. 2007. Estimation of wheat crop area in Khorasan Razavi province using TERRA satellite imagery. Agricultural Science and Technology (Soil, Water, Air), 21-2, pp.67-74.

Wardlow, D.B., L.E. Eghbert and J.H. Castens. 2007. Analysis of time-series MODIS $250 \mathrm{~m}$ vegetation index data for crop classification in the U.S. Central Great plains. J. Remote Sens. of Environ.108: 290-310.

Yan, E., Wang, G., Lin, H., Xia, C. and Sun, H., 2015. Phenology-based classification of vegetation cover types in Northeast China using MODIS NDVI and EVI time series. International Journal of Remote Sensing, 36(2), pp.489512 .

Zhang, X., Nan, Z., Sheng, Y., Zhao, L., Zhou, G., Yue, G. and Wu, J., 2010, July. Analysis of time-series modis $250 \mathrm{M}$ vegetation index data for vegetation classifiation in the wenquan area over the qinghai-tibet plateau. In 2010 IEEE International Geoscience and Remote Sensing Symposium (pp. 2059-2062). IEEE. 